PROCEEDINGS OF THE

AMERICAN MATHEMATICAL SOCIETY

Volume 130, Number 3, Pages 673-674

S 0002-9939(01)06108-1

Article electronically published on September 28, 2001

\title{
RADICALS AND PLOTKIN'S PROBLEM CONCERNING GEOMETRICALLY EQUIVALENT GROUPS
}

\author{
RÜDIGER GÖBEL AND SAHARON SHELAH
}

(Communicated by Stephen D. Smith)

\begin{abstract}
If $G$ and $X$ are groups and $N$ is a normal subgroup of $X$, then the $G$-closure of $N$ in $X$ is the normal subgroup $\bar{X}^{G}=\bigcap\{\operatorname{ker} \varphi \mid \varphi: X \rightarrow$ $G$, with $N \subseteq \operatorname{ker} \varphi\}$ of $X$. In particular, $\overline{1}^{G}=R_{G} X$ is the $G$-radical of $X$. Plotkin calls two groups $G$ and $H$ geometrically equivalent, written $G \sim H$, if for any free group $F$ of finite rank and any normal subgroup $N$ of $F$ the $G$-closure and the $H$-closure of $N$ in $F$ are the same. Quasi-identities are formulas of the form $\left(\bigwedge_{i \leq n} w_{i}=1 \rightarrow w=1\right)$ for any words $w, w_{i}(i \leq n)$ in a free group. Generally geometrically equivalent groups satisfy the same quasiidentities. Plotkin showed that nilpotent groups $G$ and $H$ satisfy the same quasi-identities if and only if $G$ and $H$ are geometrically equivalent. Hence he conjectured that this might hold for any pair of groups. We provide a counterexample.
\end{abstract}

In a series of paper, B. I. Plotkin and his collaborators [6, 3, 4, 5] investigated radicals of groups and their relation to quasi-identities. If $G$ is a group, then the $G$-radical $R_{G} X$ of a group $X$ is defined by

$$
R_{G} X=\bigcap\{\operatorname{ker} \varphi \mid \varphi: X \rightarrow G \text { any homomorphism }\} .
$$

Clearly, $R_{G} X$ is a characteristic, hence a normal subgroup of $X$. The radical $R_{G}$ can also be used to define the $G$-closure $\bar{U}^{G}=\bar{U}$ of a normal subgroup $U$ of $X$, by saying that $\bar{U} / U=R_{G}(X / U)$. This immediately leads to Plotkin's definition of geometrically equivalent groups (see [6, 3, 4, 5] and [2, p. 113]).

Definition 0.1. Let $G$ and $H$ be two groups. Then $G$ and $H$ are geometrically equivalent, written $G \sim H$, if for any free group $F$ of finite rank and any normal subgroup $U$ of $F$ the $G$ - and $H$-closures of $U$ in $F$ are the same; i.e., for any normal subgroup $U$ we have $\bar{U}^{G}=\bar{U}^{H}$.

It is easy to see that $G \sim H$ if and only if $R_{G} K=R_{H} K$ for all finitely generated groups $K$. Plotkin notes that geometrically equivalent groups satisfy the same quasi-identities. The well-known notion of quasi-identities relates to quasivarieties of groups. A quasi-identity is an expression of the form

$$
w_{1}=1 \wedge \cdots \wedge w_{n}=1 \rightarrow w=1 \text { where } w_{i}, w \in F(i \leq n) \text { are words. }
$$

Received by the editors September 6, 2000 and, in revised form, September 21, 2000.

2000 Mathematics Subject Classification. Primary 20E06, 20E10, 20E32; Secondary 20F06.

The authors were supported by project No. G 0545-173, 06/97 of the German-Israeli Foundation for Scientific Research \& Development. This paper is \#GbSh 741 in Shelah's list of publications. 
Moreover the following was shown in [6] (see [2, p.113]).

Theorem 0.2. (a) If $G \sim H$, and $G$ is torsion-free, then $H$ is torsion-free.

(b) If $G, H$ are nilpotent, then $G \sim H$ if and only if $G$ and $H$ satisfy the same quasi-identities.

This led Plotkin to conjecture that two groups might be geometrically equivalent if and only if they satisfy the same quasi-identities (see the Kourovka Notebook 2, p.113, problem 14.71]). In this note we refute this conjecture. Clearly there are only countably many finitely presented groups which we enumerate as the set $\mathfrak{K}=\left\{K_{n}: n \in w\right\}$ and let $G=\prod_{n \in w} K_{n}$ be the restricted direct product. Then $G$ satisfies only those quasi-identities satisfied by all groups and so if $H$ is any group with $G \leq H, G$ satisfies the same quasi-identities as $H$.

R. Camm [1, p. 68, p. 75 Corollary] proved there are $2^{\aleph_{0}}$ non-isomorphic, twogenerator, simple groups (see also Lyndon, Schupp [7, p. 188, Theorem 3.2]). So there exists a 2 -generated simple group $L$ which cannot be mapped nontrivially into $G$. We consider the pair $G, H=L \times G$ and show the following:

Theorem 0.3. If $G, H$ and $L$ are as above, $R_{G} L=L$ and $R_{H} L=1$. In particular $G$ and $H$ are not geometrically equivalent. Since $G \leq H$ satisfy the same quasiidentities, this is the required counterexample.

Proof. Since $L$ is a two-generated simple group, $L$ is an epimorphic image of a free group of rank 2. So it is enough to prove that $R_{G} L=L$ and $R_{H} L=1$. The first equality follows since there is no nontrivial homomorphism of $L$ into $G$. On the other hand, there is a canonical embedding $L \rightarrow H=L \times G$, so $R_{H} L=1$.

\section{REFERENCES}

[1] R. Camm, Simple free products, Journ. London Math. Soc. 28 (1953) 66-76. MR 14:616f

[2] E.I. Khukhro and V.D. Mazurov, Unsolved problems in group theory; the Kourovka Notebook, Russian Academy of Science, Novosibirsk, 1999. 13th ed. 1995 MR 97d:20001

[3] B. Plotkin, Radicals in groups, operations on classes of groups, and radical classes, Transl., II Ser. Amer. Math. Soc. 119, (1983) 89-118.

[4] B. Plotkin, Radicals and verbals, Radical theory, Colloqu. Math. Soc. Janos Bolyai 38, (1985) 379-403. MR 88f:16008

[5] B. Plotkin, Universal Algebra, Algebraic Logic, and Databases, Kluwer Acad. Publ. Dordrecht, Boston, London 1994. MR 95c:68061

[6] B. Plotkin, E. Plotkin, A. Tsurkov, Geometrical equivalence of groups, Commun. Algebra 27, (1999) 4015-4025. MR 2000e:08006

[7] R. C. Lyndon and P. E. Schupp, Combinatorial Group Theory, Springer Ergebinsberichte 89, Berlin-Heidelberg-New York, 1977. MR 58:28182

Fachbereich 6, Mathematik und Informatik, Universität Essen, 45117 Essen, Germany

E-mail address: R.Goebel@uni-essen.de

Department of Mathematics, Hebrew University, Jerusalem, Israel-And-Rutgers University, New Brunswick, New Jersey

E-mail address: Shelah@math.huji.ac.il 